\title{
Nutrition and Physical Activity Clinical Practice Guidelines for Older Adults Living with Frailty
}

\author{
A.L. Lorbergs ${ }^{1}$, J.C.Prorok ${ }^{1}$, J. Holroyd-Leduc ${ }^{2}$, D.R. Bouchard ${ }^{3}$, A. Giguere ${ }^{4}$, L. Gramlich ${ }^{5}$, H. Keller ${ }^{6}$, A. Tang ${ }^{7}$, \\ M. Racey ${ }^{8}$, M.U.Ali ${ }^{9}$, D. Fitzpatrick-Lewis ${ }^{10}$, D. Sherifali ${ }^{8}$, P. Kim ${ }^{1}$, J. Muscedere ${ }^{1,11}$
}

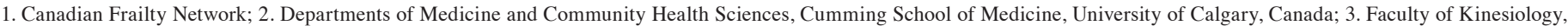

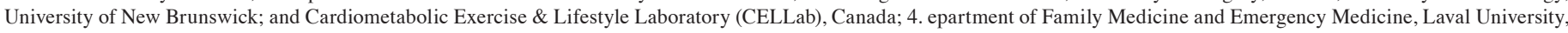

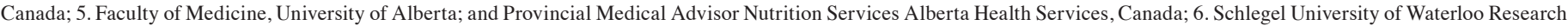

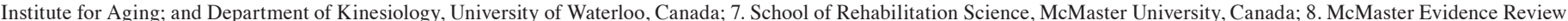

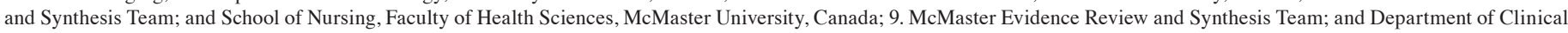

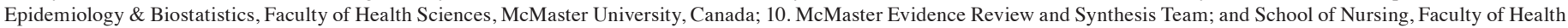
Sciences, McMaster University, Canada; 11. Department of Critical Care Medicine, Queens University, Canada

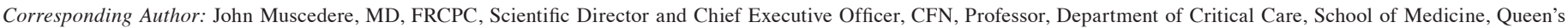
University, Canada, John.Muscedere@kingstonhsc.ca

\begin{abstract}
BACKGROUND: We developed clinical practice guidelines to provide health care providers with evidence-based recommendations for decisions related to the effective management of frailty and pre-frailty using nutrition and physical activity interventions.

METHODS: We based the recommendations on two systematic reviews with meta-analyses. Nutrition, physical activity, and combined nutrition and physical activity interventions for adults $\geq 65 \mathrm{y}$ were considered if study populations were identified as frail using a frailty tool or assessment. Risk of bias and certainty of evidence were evaluated. We included physical outcomes, mobility, frailty, cognitive function, activities of daily living, falls, quality of life, diet quality, energy/fatigue levels, health services use, and caregiver outcomes.

RESULTS: Overall, mobility improvements were moderate with nutritional strategies that optimize dietary intake, various types of physical activity interventions, and interventions that combine nutrition and physical activity. Physical outcomes, such as body mass and muscle strength, improved moderately with nutritional strategies and interventions that combined nutrition with physical activity. Frailty status improved with multi-component physical activity interventions. Strong recommendations include optimizing dietary intake, performing physical activity, and adopting interventions that combine nutrition and physical activity. We strongly recommend various types of physical activity including muscle strengthening activities, mobilization or rehabilitation exercises, and multi-component physical activity interventions.

INTERPRETATION: Tailored nutrition and physical activity interventions based on individual goals and health status are associated with improved clinical and physical outcomes. While the recommendations facilitate shared decision-making, we identified sparse application of validated frailty assessments and lack of standardized research outcomes as critical gaps in knowledge.
\end{abstract}

Key words: Shared decision making, frailty, guidelines, nutrition, exercise.

\section{Introduction}

$\mathrm{O}$ Ider adults are disproportionately affected by frailty, defined as an increased vulnerability to negative health outcomes resulting from decline in reserve and function across multiple physiologic systems $(1,2)$. In Canada, it is estimated that 1.5 million people are frail, which may grow to well over two million Canadians within the next 10 years (3). Frailty is not an inevitable part of aging, although it is considered a major public health concern because it increases the risk for negative health outcomes, including falls, delirium, hospitalization, functional dependency, admission to continuing care and death (4-8). Frailty is a dynamic state which can improve, but more commonly can worsen (9). Conversely, pre-frailty is considered a multi-dimensional and potentially reversible risk state that is present before frailty develops (10). While both older adults with frailty and prefrailty could be relatively independent those with frailty face a greater number of health deficits and have less capacity to resist negative consequences of stressors compared to those with pre-frailty. Since the ability to reduce frailty severity is more likely in its early stages, preventing frailty in the first place or intervening when pre-frailty is present are considered the most effective means of preserving older adults' independence and quality of life $(9,11)$.

Weight loss and low physical activity, among other factors such as social support and cognitive function, are important contributors to frailty and its progression, primarily through bone, muscle, tolerance, and cardiovascular health implications. The management of older adults with frailty and pre-frailty with a holistic care approach, including nutrition and physical activity interventions, can reduce the risk of adverse health outcomes (12). Although the benefits of nutritional and physical activity interventions for older adults with frailty have been described $(13,14)$, their effectiveness on frailty severity and important health outcomes have not been synthesized for use by health care providers. Physical activity guidelines are available for healthy older adults (15); however, there are no 
clinical practice guidelines (CPGs) that provide evidencebased recommendations about nutrition and physical activity specifically for older adults living with frailty and pre-frailty. Targeted nutrition and physical activity guidelines for this population are important because of the specific care needs associated with frailty, complex comorbidities, and functional loss. The CPGs presented in this paper are evidence-based and can be incorporated into the care plans of older adults with frailty or pre-frailty along with consideration of the individual's health, energy needs, and goals (16). These newly developed nutrition and physical activity CPGs for older adults living with frailty and pre-frailty fill a critical clinical practice gap, as well as identify avenues for further research.

The objective of the CPGs is to provide health care professionals with evidence-based recommendations for the management of frailty and pre-frailty using nutrition and physical activity interventions as part of a holistic care plan. The target population is individuals aged 65 years and older, who have been assessed and identified as frail or pre-frail, and who may be residing in community or continuing care settings.

\section{Methods}

The development of these guidelines followed the Appraisal of Guidelines, Research and Evaluation (AGREE II) framework (17) and is reported according to the AGREE Reporting Checklist (18). An interdisciplinary CPG Steering Committee (See Appendix A for membership) was established in 2018 consisting of experts from across Canada with clinical and content expertise in frailty, aging, nutrition, physical activity, and knowledge translation. The Steering Committee met regularly in-person and via teleconference to establish the guideline priorities and develop the research questions based on a population, intervention, comparator, and outcomes (PICO) format. In partnership with MERST (McMaster Evidence Review and Synthesis Team), we conducted two systematic reviews to determine the effectiveness of: 1) nutrition and combined nutrition and physical activity interventions, and 2) physical activity interventions for older adults with frailty and pre-frailty $(19,20)$. We included studies with participants aged 65 years and older who were identified as frail or pre-frail using a frailty tool, or other explicit author-established frailty criteria. The harms and health outcomes of interest were selected, broadly grouped, and their importance determined according to a majority voting process by the Steering Committee. We included clinical outcomes (e.g., frailty as measured by a valid tool), self-reported outcomes (e.g., quality of life as measure by a standardized tool), health services use (e.g., hospitalization), and caregiver outcomes (e.g., caregiver burden). See Appendix B for a complete list of outcomes for each systematic review.
Three external content and methods experts provided blinded reviews of the research questions, literature search protocols, and analytic framework for the systematic reviews. The protocols were registered in PROSPERO (CRD42020144819; CRD42020144556) and are available at https://www.crd.york. ac.uk/prospero/.

We performed literature searches for each PICO question in four electronic databases (MEDLINE, EMBASE, Cochrane, and CINAHL). Only English language published literature was included from database inception up to July 2019. Full search strategies are presented in Appendix C. All nutrition and combined interventions, including randomized and quasiexperimental designs, with a control group were considered. Combined approach interventions had to include both nutrition and physical activity components (but no other type of intervention such as memory tasks) in the same intervention, as compared to a control group. All physical activity interventions were considered, including those performed independently, supervised, assisted, or in a group setting. Respiratory training was excluded from physical activity interventions. For all studies, a control group was defined as usual care, routine care, or minimal contact that did not include any intervention or treatment group components. For all outcomes, the effects of interventions were compared to minimal contact intervention or usual care. Where possible, unadjusted intention-to-treat data were extracted and assessment of risk of bias was done using the Cochrane Collaboration risk-of-bias tool (21) for randomized control trials (RCTs). Papers were graded for confidence in the effect and assigned a level of confidence using the GRADE (Grading of Recommendations, Assessment, Development, and Evaluation) system (22) whereby evidence is assessed at 1 of 4 confidence levels (very low, low, moderate, or high) based on specific criteria (Table 1). We developed evidence to decision (EtD) tables to summarize the evidence for all critical outcomes, explicitly record judgements made during data interpretation, and determine the direction and strength of recommendations. For the full search strategy and methods description refer to the evidence reviews $(19,20)$.

For each recommendation, judgements were made about the net benefit of the intervention, indicating whether it was positive, negative, or uncertain. Strong recommendations indicate that the intervention is likely to have benefits that outweigh any risk, and that most health care providers would prescribe this intervention (23). Strong recommendations may not apply to all persons in all conditions since no recommendation can consider all the individual circumstances. Weak (i.e., conditional) recommendations imply that whilst some health care providers would prescribe the intervention, some would not because of the balance between harms and benefits of the intervention (24). The Steering Committee

Table 1. GRADE Ranking of Evidence

High Further research is very unlikely to change our confidence in the estimate of effect

Moderate Further research is likely to have an important impact on the confidence in the estimate of effect and may change the estimate

Low Further research is very likely to have an important impact on our confidence in the estimate of effect and is likely to change the estimate

Very Low Any estimate of the effect is very uncertain 
Table 2. Key recommendations for older adults with frailty or pre-frailty

\begin{tabular}{|l|l|l|}
\hline Strong Recommendations & Nutrition & Physical Activity \\
& $\begin{array}{l}\text { We recommend that older adults who are living with frailty or pre-frailty adopt combined physical activity and } \\
\text { nutrition strategies. } \\
\text { We recommend nutritional strategies to optimize dietary } \\
\text { intake in older adults living with frailty and pre-frailty. }\end{array}$ & $\begin{array}{l}\text { We recommend that older adults living with frailty or } \\
\text { pre-frailty perform muscle strengthening activities. } \\
\text { We recommend that older adults living with frailty or } \\
\text { pre-frailty perform physical activity. } \\
\text { We recommend that older adults living with frailty or pre- } \\
\text { frailty perform mobilization or rehabilitation exercises. }\end{array}$ \\
& $\begin{array}{l}\text { We recommend that older adults living with frailty or } \\
\text { pre-frailty perform multi-component physical activity } \\
\text { (i.e., incorporating combinations of aerobic, resistance, } \\
\text { balance, and flexibility training) }\end{array}$ & $\begin{array}{l}\text { We suggest that older adults living with frailty or pre- } \\
\text { frailty perform aerobic physical activity. }\end{array}$ \\
\hline Weak Recommendations & $\begin{array}{l}\text { We suggest that older adults living with frailty or pre- } \\
\text { frailty consume protein fortified foods/supplements. }\end{array}$ &
\end{tabular}

developed recommendations based on the highest level of evidence and judgements from the EtD tables, as well as through consensus. Following drafting of recommendations, we invited key stakeholders to participate in a full-day virtual stakeholder meeting to discuss the draft recommendations. Stakeholders from across Canada included: family physicians, physiotherapists, nurses, geriatricians, kinesiologists, members of stakeholder organizations (e.g., Dietitians of Canada) and content experts (See Appendix D for a complete list of participants). All participants in the guideline development disclosed actual and potential conflicts of interest using a standardized reporting form. Those who had competing interests were not restricted in their participation but were asked to abstain where conflict could be perceived.

\section{Recommendations}

We developed eight CPG statements using best available evidence and expert consensus (Table 2). The guidelines may be applied across care settings with consideration for the older adults' goals and practitioner expertise.

\section{Recommendation 1: We recommend nutritional strategies to optimize dietary intake in older adults living with frailty and pre-frailty [strong recommendation; low certainty of evidence]}

This recommendation was based on eight RCTs comprised of a total of 1071 individuals (25-32). The interventions included: vitamin D supplementation (26), protein supplementation $(28,30,31)$, diet supplementation with a commercial formulation $(27,29)$, and dietary modification to enhance nutrient density $(25,32)$. Overall, there was a positive effect of moderate certainty on frailty (SMD: $-0.22,95 \% \mathrm{CI}$ : -0.44 to $-0.01 ; 3$ studies: $n=255$ ), physical outcomes (e.g., activities of daily living, muscle strength, appendicular lean mass) (SMD: 0.16; 95\% CI: 0.02 to $0.29 ; 7$ studies: $n=694$ ), and mobility (e.g., gait speed, timed up and go, sit to stand test, balance, short physical performance battery) (SMD: 0.15,
95\% CI: 0.001 to $0.3 ; 7$ studies: $n=694$ ) (20). There was no significant effect on body weight, body mass index (SMD: $-0.18,95 \% \mathrm{CI}$ : -0.51 to 0.16 ; 4 studies: $n=284$ ) and quality of life (SMD: $-0.12,95 \%$ CI: -1.39 to $1.15 ; 1$ study, $n=243$ ). The impact on caloric intake was reported in five studies as assessed at 12 to 24 weeks $(25,27,28,31,32)$; however, there was no statistically significant increase in the intake of calories per day. There was serious risk of bias for all outcomes, except quality of life, but no harms or adverse events were identified.

\section{Recommendation 2: We suggest that older adults living with frailty or pre-frailty consume protein fortified foods/supplements. [weak recommendation; low certainty of evidence]}

We considered five RCTs comprised of a total 344 individuals $(27,28,30-32)$. The amount and type of protein supplementations in four of the included studies were: $25 \mathrm{~g}$ protein/day (27), 30g protein/day (28), $24 \mathrm{~g}$ whey protein/day (30), and $16 \mathrm{~g}$ soy protein/day (32). The fifth study assigned the intervention group $1.2 \mathrm{~g} / \mathrm{kg}$ of protein to $1.5 \mathrm{~g} / \mathrm{kg}$ of protein/ day; however, the amount of additional protein was not reported (31). The protein content of diets in the control groups ranged from $0.8 \mathrm{~g} / \mathrm{kg} /$ day to $1.05 \mathrm{~g} / \mathrm{kg} /$ day; this range is potentially inadequate to support improvements in frailty and other outcomes.

Overall, there was a positive effect of moderate certainty for physical outcomes (SMD: 0.16; 95\% CI: 0.01 to $0.31 ; 5$ studies: $\mathrm{n}=344$ ) and mobility (SMD: $0.20,95 \%$ CI: 0.02 to 0.39 ; 5 studies: $\mathrm{n}=344)$ (20). There was no statistically significant difference in body weight, body mass, frailty, or caloric intake. All studies had a serious risk of bias, but no harms or adverse events. 
Recommendation 3: We recommend that older adults living with frailty or pre-frailty perform physical activity [strong recommendation; moderate certainty of evidence]

We considered twenty-six studies (20 RCTs, three quasiexperimental trials, two prospective cohort studies, one prospective controlled clinical trial) with a total enrolment of 8022 participants for this recommendation $(25,29,33-56)$. The studies included had several types of physical activity interventions, including aerobic activity, muscle strengthening, mobilization/rehabilitation, and multi-component physical activity. Moderate certainty evidence suggests that physical activity reduces older adults' level of frailty (SMD: -1.29, 95\% CI: -2.22 to -0.36 ; 4 studies: $n=244$ ) (19). This finding is strengthened by additional moderate certainty evidence suggesting that physical activity reverses frailty (RR: $0.58,95 \%$ CI: 0.36 to 0.93 ; 4 studies: $\mathrm{n}=2050$ ).

Moderate certainty evidence also suggests that physical activity improves mobility (SMD: $0.60,95 \% \mathrm{CI}: 0.37$ to 0.83 ; 19 studies: $\mathrm{n}=1724$ ), activities of daily living (SMD: 0.50, 95\% CI: 0.15 to $0.84 ; 9$ studies: $n=910$ ), and quality of life (SMD $0.60,95 \%$ CI: 0.13 to 1.07 ; 6 studies: $\mathrm{n}=500$ ) of older adults living with frailty or pre-frailty (19). Additionally, moderate certainty evidence suggests that physical activity has a small effect on cognitive functions (SMD: $0.35,95 \%$ CI: 0.09 to $-0.61 ; 5$ studies: $\mathrm{n}=377$ ).

Low certainty evidence suggests that physical activity makes little to no difference in the fatigue level experienced by older adults living with frailty or pre-frailty (SMD: $-0.27,95 \%$ CI: -0.65 to 0.12 ). Very low certainty evidence suggests there is no effect of physical activity on older adults' risks of falling (RR: $0.80,95 \%$ CI: 0.51 to 1.26 ; 7 studies: $\mathrm{n}=724$ ). Potential harms of physical activities were included in 12 of the 26 included studies. None of the harms were serious or significantly different compared with the control group.

It is uncertain whether one type of physical activity is superior to others for improving clinical, self-reported, or health services use outcomes because the relative effectiveness of the different types of physical activity was not evaluated.

Recommendation 4: We recommend that older adults living with frailty or pre-frailty perform muscle strengthening activities [strong recommendation; moderate certainty of evidence]

We considered eight studies comprising a total of 1058 individuals $(33,36,39,40,44,46,50,52)$. Five of these studies were RCTs and three were quasi-experimental trials. Moderate certainty evidence suggests that muscle strengthening improves mobility (SMD: $0.57,95 \%$ CI: 0.08 to 1.06 ; 6 studies: $\mathrm{n}=722$ ) and reverses frailty severity (RR: $0.21,95 \%$ CI: 0.10 to 0.43 ; 1 study: $n=66$ ) (19). However, very low certainty evidence reported no effect of muscle strengthening on frailty severity in older adults (SMD: $-0.20,95 \%$ CI: -0.79 to 0.39 ;
1 study: $\mathrm{n}=45$ ). Low certainty evidence suggests that muscle strengthening activities may make little or no difference to activities of daily living (SMD: $0.16,95 \%$ CI: -0.05 to 0.37 ; 2 studies: $n=278$ ). There is very low certainty evidence on the effects of muscle strengthening activities on cognitive function (SMD: $0.45,95 \%$ CI: 0.19 to $0.72 ; 1$ study: $n=45$ ), quality of life (SMD: $0.15,95 \%$ CI: -0.33 to $0.63 ; 1$ study: $n=70$ ), or risks of falling (RR: $0.78,95 \%$ CI: 0.37 to $1.65 ; 2$ studies: $n=442$ ) (19). We did not find any evidence on the effectiveness of muscle strengthening activities on fatigue level and hospital service use.

The findings demonstrate that some elements of mobility would be improved by muscle strengthening. Yet, most studies reported non-significant improvements in comprehensive scores of mobility, such as the short physical performance battery.

Five studies did not report adverse effects of muscle strengthening. Of the four that studied adverse effects, a single study (36) reported falls and admissions to the hospital or care homes with no significant difference between the intervention and the control groups.

\section{Recommendation 5: We recommend that older adults living with frailty or pre-frailty perform mobilization or rehabilitation exercises [strong recommendation; moderate certainty of evidence]}

Mobilization and rehabilitation exercises were defined as low-intensity physical activities that challenge balance, flexibility, coordination, and may incorporate technical training to facilitate safe movement. We considered four RCTs with a total enrolment of 478 individuals for this recommendation $(48,49,53,56)$. Moderate certainty of evidence suggests that mobilization or rehabilitation exercises may improve mobility (SMD: $0.29,95 \%$ CI: 0.17 to $0.42 ; 3$ studies: $\mathrm{n}=330$ ) and activities of daily living (SMD 0.48; 95\% CI: 0.28 to 0.67 ; 1 study: $\mathrm{n}=182$ ) (19). Low certainty evidence suggests that mobilization or rehabilitation exercises have no effect on cognitive function (SMD: $0.12,95 \%$ CI: -0.10 to 0.34 ; 1 study; $\mathrm{n}=116$ ) or risks of falling (RR: $0.88,95 \%$ CI: 0.69 to $1.12 ; 1$ study; $\mathrm{n}=184$ ).

Mobilization and rehabilitative exercise interventions seem to be safe. One study (48) reported adverse events. Angina cases were higher in the intervention group, but the rates of other adverse events (e.g., falls, musculoskeletal problems, chest pain, death) did not differ significantly between the intervention and control groups. 
Recommendation 6: We recommend that older adults living with frailty or pre-frailty perform multi-component physical activity (i.e., incorporating combinations of aerobic, resistance, balance, and flexibility training) [strong recommendation; moderate certainty of evidence]

This recommendation was based on 11 RCTs comprised of 2646 participants $(25,29,34,35,38,41,43,45,47,54,55)$. Moderate certainty evidence suggests that multi-component physical activity interventions have a large effect on reducing frailty severity (SMD: $-1.57,95 \%$ CI: -2.57 to $-0.57 ; 3$ studies: $\mathrm{n}=199$ ) (19). This finding is strengthened by moderate certainty evidence that multi-component physical activity can reverse frailty (RR: $0.72,95 \%$ CI: 0.63 to $0.83 ; 3$ studies: $\mathrm{n}=1472$ ). Moderate certainty evidence also suggests that multicomponent physical activity interventions have a medium effect size in improvements in mobility (SMD: $0.75,95 \%$ CI: 0.40 to 1.10; 9 studies: $\mathrm{n}=636$ ) (19).

Similarly, moderate certainty evidence suggests that multicomponent physical activity have medium-sized effects on improving cognitive function (SMD: $0.62,95 \%$ CI: 0.12 to 1.11 ; 2 studies: $\mathrm{n}=180$ ) and quality of life (SMD: $0.68,95 \% \mathrm{CI}$ : 0.16 to $1.21 ; 5$ studies: $\mathrm{n}=430$ ) (19). Low certainty evidence suggests that multi-component physical activity interventions may improve performance of activities of daily living of older adults living with frailty or pre-frailty (SMD: $0.64,95 \% \mathrm{CI}$ : 0.004 to $1.27 ; 5$ studies: $\mathrm{n}=414$ ).

However, multi-component physical activity interventions may make little or no difference to older adults' risks of falling or health services use. Low certainty evidence suggests that multi-component physical activity had no effect on number of falls (SMD: $-0.37,95 \%$ CI: -0.81 to 0.07 ), risk of falling (RR: $0.62,95 \%$ CI 0.16 to 2.47 ), emergency visits (SMD: $-0.21,95 \%$ CI: -0.65 to 0.23 ), or risk of hospitalization (RR: $0.52,95 \%$ CI: 0.05 to 5.56$)$ (19). It remains uncertain whether older adults living with frailty or pre-frailty should perform multi-component physical activity interventions to reduce their fatigue level. Very low certainty evidence suggests that multicomponent physical activity reduces fatigue levels (SMD: $-0.23,95 \%$ CI: -0.85 to $0.39 ; 2$ studies: $\mathrm{n}=148$ ).

Many studies did not report adverse events. Minor events, such as pain, were managed by adjusting the training program. We suggest that consideration be placed on the presence of comorbidities that may affect participation, such as arthritis or other causes of joint pain.

Recommendation 7: We suggest that older adults living with frailty or pre-frailty perform aerobic physical activity [weak recommendation; low certainty of evidence]

This recommendation was based on one RCT that studied 36 community dwelling individuals with frailty (42). Low certainty evidence suggests that aerobic physical activity may improve mobility of older adults living with frailty or pre-frailty (SMD: 0.71, 95\% CI: 0.23 to 1.20 ) (19). It is uncertain whether aerobic physical activity improves the activities of daily living, cognitive function, or fatigue of older adults living with frailty or pre-frailty. Indeed, very low certainty evidence suggests that aerobic physical activity has no effect on activities of daily living (SMD: $0.46,95 \%$ CI: -0.03 to 0.94 ), cognitive function (SMD: $0.15,95 \%$ CI: -0.50 to 0.80 ), or fatigue (SMD: -0.39 , 95\% CI: -0.87 to 0.09$)$ (19).

The harms of aerobic activity for older adults with frailty are unknown because they were not reported in the included study (42). We found no evidence related to aerobic activity interventions for older adults in continuing care settings.

Recommendation 8: We recommend that older adults who are living with frailty or pre-frailty adopt combined physical activity and nutrition strategies [strong recommendation; low certainty of evidence]

This recommendation was based on five RCTs $(25,43$, 57-59), two case-control trials $(60,61)$ and one pilot trial (62) with a total enrolment of 971 participants. Diet counselling was used as the nutrition intervention in four studies: faceto-face $(57,58)$, via telephone $(61)$, or with cooking classes (43). Four studies included fortified/enhanced foods including whey protein $(60)$ or supplements $(25,28,59,62)$. The role of micronutrient (i.e., vitamin) supplementation was not addressed by these studies. Six studies focused their physical activity interventions on muscle strengthening $(43,58-62)$, while others used a multi-component approach $(25,57)$.

There was a positive effect of moderate certainty for physical outcomes (SMD: 0.19, 95\%CI: 0.06 to $0.32 ; 6$ studies: $\mathrm{n}=514$ ) and mobility (SMD: 0.25, 95\%CI: 0.02, 0.48; 6 studies: $\mathrm{n}=514$ ) (20). Frailty severity was reduced in five studies of moderate certainty. There was no effect on diet quality, but this was of very low certainty due to inconsistency and imprecision of diet assessment. In the three studies that reported quality of life there was no effect, and the certainty of evidence was low (20).

Combined physical activity and nutrition interventions seem to be safe. The most common adverse event reported by the intervention arm participants was back pain. There was serious risk of bias across all studies.

\section{Discussion}

These evidence-informed CPGs provide recommendations for the use of both nutrition and physical activity to improve physical functioning in older adults with frailty and pre-frailty. Nutrition and physical activity are evidence-based lifestyle factors that can be modified by an individual, with proper support from social or healthcare systems, to help reduce frailty severity. Notwithstanding, older adults with frailty and prefrailty are a special population that, depending on the severity of frailty, may present to a health practitioner with complex needs, which would need to be identified and addressed prior 
to initiating a nutrition and/or physical activity program. The recommendations presented in this document are intended to be interpreted and modified according to an older adult's goals, functioning, care setting, and the clinical expertise of the care provider. To maximize the effectiveness of nutrition and physical activity interventions for frail and pre-frail older adults, important factors to assess and consider are malnutrition status, physical disabilities, cognition, and environmental barriers.

Insufficient evidence was available to understand the impact of nutrition and physical activity interventions on important outcomes such as mortality, quality of life, caregiver burden, and health services utilization. Nevertheless, the CPGs suggest benefits of nutrition and physical activity interventions to physical outcomes, mobility, and frailty status. Thus, we suggest that primary care physicians consider referrals to dietitians or community nutrition education programs where available to support tailoring, as well as individualized support for behaviour change. We also encourage physicians and other health care providers to consider using physical activity prescriptions that consider an older adult's level of frailty at the time of assessment. The activity prescription should indicate appropriate activity types (e.g., muscle strengthening, mobilization, aerobic) and be tailored to an individual's functional status, activity preferences, and cognitive status. Referrals to exercise experts in the community (e.g., certified exercise physiologists, kinesiologists, physiotherapists) and integration of behaviour change strategies, such as motivational interviewing, may be appropriate to ensure tailored support.

These CPGs will be hosted on publicly accessible websites accompanied by a lay summary of the recommendations and their implications for older adults with frailty and their families. They will be disseminated through professional associations and organizations advocating for older adults using their webbased newsletters, social media posts, and education resources for health professionals to increase awareness. Implementation of the CPGs will be facilitated by consulting with relevant stakeholders, including older adults and their family members. Clear language tools for older adults may be developed to incorporate local nutrition and physical activity resources and supports for older adults.

There are several system-level and person-oriented barriers to implementation of the CPGs. One key barrier is the limited recognition of frailty as a health condition by many provincial and territorial health systems. This prevents physicians from systematically recognizing an older adult's frailty status, which leads to missed opportunities in shaping and managing care goals. Depending on the type of referral that health care providers make it is worth acknowledging that barriers such as cost to the health care system and patient, resource availability (e.g., dietitians, exercise specialists, physiatry), and social determinants of health (e.g., ethnicity, poverty, housing, family supports) may preclude successful intervention. Other barriers to initiating a physical activity intervention from perspective of older adults include lack of motivation, limited access to transportation, and increased caregiver burden (63). Persons with more severe frailty, comorbid conditions and/or associated pain and functional limitations may experience complex physical challenges. Potential risks of initiating exercise when nutrition is inadequate include weight loss, limited exercise capacity, or attenuated muscle strength gains. From a health care provider perspective implementing a physical activity program in clinical practice, barriers include inadequate planning and coordination among health care professionals and uncertainty about the safety of referring persons with frailty/ pre-frailty to community programs $(63,64)$. Moreover, health care providers cite limited resources, limited infrastructure, and insufficient time to discuss specifics about an exercise program with older adults (65). Tailored implementation strategies are needed to encourage the uptake of nutrition and physical activity CPGs.

\section{Other Guidelines}

In 2019 an international task force published CPGs for the identification and management of frailty (66) and recommended that health practitioners use validated assessment tools to identify frailty among older adults. For those identified as frail, a comprehensive care plan that addresses polypharmacy and causes of exhaustion is recommended. In line with our recommendations, the task force recommended that individuals with frailty and pre-frailty be prescribed a multi-component physical activity intervention with resistance training as first line therapy (66). They also made conditional recommendations for nutrition interventions based on very low to low certainty of evidence. However, an important distinction between our CPGs and those published by the international task force is the rigorous process we used to gather, analyze, and synthesize the best-available evidence related to physical activity and nutrition. The task force did not base their recommendations on a formal assessment of effectiveness and systematic review of biases. In contrast, our recommendations are based on two systematic reviews and meta-analyses that were specifically conducted for the development of these CPGs. We included clearly identified frail and pre-frail participants and looked at the effectiveness of nutrition, physical activity, and combined nutrition and physical activity interventions on a more robust set of outcomes.

The Canadian 24-Hour Movement Guidelines (24HMGs) for adults aged $18-64 \mathrm{y}$ and adults aged $65 \mathrm{y}$ and older were recently developed to set behavioural benchmarks and inform public health messaging (15). The 24HMGs provide physical activity, as well as sleep and sedentary behaviour recommendations to improve health outcomes. It is recommended that adults aged $65 \mathrm{y}$ and older should aim to accumulate at least 150 minutes per week of moderate to vigorous physical activity, muscle strengthening activities at least twice a week, and several hours of light physical activities, including standing (15). It is also recommended that adults aged $65 \mathrm{y}$ and older include physical activities that challenge balance. Despite using best available evidence, the $24 \mathrm{HMG}$ s exclude older adults experiencing frailty and those living in continuing care. Nevertheless, key strengths of the 24HMGs are the explicit inclusion of sedentary behaviour and a collaborative and systematic approach to knowledge translation efforts (67). 


\section{Gaps in Knowledge}

There is limited high certainty evidence regarding nutrition and physical activity interventions for the management of frailty and pre-frailty. The scarcity of such evidence can be attributed to the limited use of frailty assessment in research studies, as well as a lack of standardized research outcomes for frailty research. First, both researchers and health care providers are challenged by the lack of uniformly agreed-upon criteria for operationalizing frailty. However, it is important to recognize frailty, independent of chronological age, chronic diseases, and disability, so that it can be managed appropriately (68). We strongly recommend that future studies include frailty assessment of older participants, both for targeting the intervention and as an outcome, to bolster the current scarcity of evidence. Next, determining a set of common outcomes for frailty research is warranted to improve the strength of the evidence and mitigate challenges associated with the analysis and interpretation of evidence to better inform care for older adults. For example, many of our recommendations would be strengthened by consistent reporting of outcomes within RCTs. Therefore, we strongly recommend that the research community prioritizes establishment of common outcomes by seeking input from clinicians, researchers, older adults living with frailty, and their caregivers to continue development of meaningful research (69). Finally, there is little evidence to demonstrate that these interventions are effective once implemented in complex, real life conditions. Future research that assesses nutrition and physical activity interventions with a pragmatic perspective is warranted.

\section{Conclusion}

The goals of frailty prevention and management are to promote mobility, mitigate adverse health outcomes, and improve quality of life among older adults. These evidenceinformed CPGs provide recommendations for the use of both nutrition and physical activity to improve physical functioning in older adults with frailty and pre-frailty. Dissemination and implementation of these CPGs will be integral components for improving the care of Canadians living with frailty. More research is required on how to prevent or mitigate frailty with evidence informed nutrition and activity-based interventions.

Acknowledgements: The authors thank the guideline panel members for their contributions to the development of these guidelines.

Funding Statement: These guidelines were supported by the Canadian Frailty Network (CFN), which is supported by the Government of Canada through the Networks of Centres of Excellence (NCE) program, as well as the McMaster Institute for Research and Aging (MIRA) and the McMaster Evidence Review and Synthesis Team (MERST). CFN was responsible for the conception and design of the project. MERST was independently responsible for the data extraction, analysis, interpretation, and reporting, which determined the content of the guidelines. CFN prepared, reviewed, and provided final approval of the manuscript. MERST supported editing of the final manuscript. Final decisions regarding the content, protocol, and issues that arose during the guideline-development process were solely the responsibility of the guideline Steering Committee.

Competing Interests: John Muscedere is the Scientific Director of the Canadian Frailty Network, which provided funding for the development of the guidelines. He did not receive payment for work on the guidelines. It is noted that four of the authors are affiliated with one of the funders of the study; however, their involvement helped inform the project design and preparation of the manuscript. CFN was not involved with data extraction or analysis. All screening of articles, data extraction, analysis, and interpretation was conducted by MERST personnel, independent from the Steering Committee or funders In forming the interdisciplinary Steering Committee and other project team members, CFN collected and reviewed Conflict of Interest forms. Heather Keller receives speaker honoraria, matching research funding, and consultation travel expenses from Abbott Nutrition, as well as speaker honoraria from Nestle Health Sciences. She did not receive payment for any aspect of the submitted work. No other competing interests were declared.

Open Access: This article is distributed under the terms of the Creative Commons Attribution 4.0 International License (http://creativecommons.org/licenses/by/4.0/), which permits use, duplication, adaptation, distribution and reproduction in any medium or format, as long as you give appropriate credit to the original author(s) and the source, provide a link to the Creative Commons license and indicate if changes were made.

\section{References}

1. Bergman H, Ferrucci L, Guralnik J, Hogan DB, Hummel S, Karunananthan S, et al. Frailty: an emerging research and clinical paradigm--issues and controversies. J Gerontol A Biol Sci Med Sci. 2007;62(7):731-7. DOI: 10.1093/gerona/62.7.731

2. Abellan van Kan G, Rolland Y, Bergman H, Morley JE, Kritchevsky SB, Vellas B The I.A.N.A Task Force on frailty assessment of older people in clinical practice. J Nutr Health Aging. 2008;12(1):29-37. DOI: 10.1007/BF02982161

3. Hoover M, Rotermann M, Sanmartin C, Bernier J. Validation of an index to estimate the prevalence of frailty among community-dwelling seniors. Health Rep. 2013;24(9):10-7.

4. Fried LP, Tangen CM, Walston J, Newman AB, Hirsch C, Gottdiener J, et al . Frailty in older adults: evidence for a phenotype. J Gerontol A Biol Sci Med Sci. 2001;56(3):M146-56. DOI: 10.1093/gerona/56.3.m146

5. Gill TM, Gahbauer EA, Han L, Allore HG. Trajectories of disability in the last year of life. N Engl J Med. 2010;362(13):1173-80. DOI: 10.1056/NEJMoa0909087

6. Ensrud KE, Ewing SK, Taylor BC, Fink HA, Cawthon PM, Stone KL, et al. Comparison of 2 frailty indexes for prediction of falls, disability, fractures, and death in older women. Arch Intern Med. 2008;168(4):382-9. DOI: 10.1001/ archinternmed.2007.113

7. Rockwood K, Howlett SE, MacKnight C, Beattie BL, Bergman H, Hebert R, et al Prevalence, attributes, and outcomes of fitness and frailty in community-dwelling older adults: report from the Canadian study of health and aging. J Gerontol A Biol Sci Med Sci. 2004;59(12):1310-7. DOI: 10.1093/gerona/59.12.1310

8. Vermeiren S, Vella-Azzopardi R, Beckwee D, Habbig AK, Scafoglieri A, Jansen B, et al. Frailty and the Prediction of Negative Health Outcomes: A Meta-Analysis. J Am Med Dir Assoc. 2016;17(12):1163 e1- e17. DOI: 10.1016/j.jamda.2016.09.010

9. Gill TM, Gahbauer EA, Allore HG, Han L. Transitions between frailty states among community-living older persons. Arch Intern Med. 2006;166(4):418-23. DOI: 10.1001/archinte.166.4.418

10. Sezgin D, Liew A, O’Donovan MR, O'Caoimh R. Pre-frailty as a multi-dimensional construct: A systematic review of definitions in the scientific literature. Geriatr Nurs. 2020;41(2):139-46. DOI: 10.1016/j.gerinurse.2019.08.004

11. Rockwood K, Mitnitski A. Frailty in relation to the accumulation of deficits. J Gerontol A Biol Sci Med Sci. 2007;62(7):722-7. DOI: 10.1093/gerona/62.7.722

12. Apostolo J, Cooke R, Bobrowicz-Campos E, Santana S, Marcucci M, Cano A, et al. Effectiveness of interventions to prevent pre-frailty and frailty progression in older adults: a systematic review. JBI Database System Rev Implement Rep. 2018;16(1):140-232. DOI: 10.11124/JBISRIR-2017-003382

13. Negm AM, Kennedy CC, Thabane L, Veroniki AA, Adachi JD, Richardson J, et al. Management of Frailty: A Systematic Review and Network Meta-analysis of Randomized Controlled Trials. J Am Med Dir Assoc. 2019;20(10):1190-8. DOI: 10.1016/j.jamda.2019.08.009

14. O'Connell ML, Coppinger T, McCarthy AL. The role of nutrition and physical activity in frailty: A review. Clin Nutr ESPEN. 2020;35:1-11. DOI: 10.1016/j. clnesp.2019.11.003

15. Ross R, Chaput JP, Giangregorio LM, Janssen I, Saunders TJ, Kho ME, et al Canadian 24-Hour Movement Guidelines for Adults aged 18-64 years and Adults aged 65 years or older: an integration of physical activity, sedentary behaviour, and sleep. Appl Physiol Nutr Metab. 2020;45(10 (Suppl. 2)):S57-S102. DOI: 10.1139/ apnm-2020-0467

16. Lee PG, Jackson EA, Richardson CR. Exercise Prescriptions in Older Adults. Am Fam Physician. 2017;95(7):425-32.

17. Brouwers MC, Kho ME, Browman GP, Burgers JS, Cluzeau F, Feder G, et al AGREE II: advancing guideline development, reporting and evaluation in health care. CMAJ. 2010;182(18):E839-42. DOI: 10.1503/cmaj.090449

18. Brouwers MC, Kerkvliet K, Spithoff K, Consortium ANS. The AGREE Reporting Checklist: a tool to improve reporting of clinical practice guidelines. BMJ 2016;352:i1152. DOI: 10.1136/bmj.i1152

19. Racey M, Ali MU, Sherifali D, Fitzpatrick-Lewis D, Lewis R, Jovkovic M, et al. Effectiveness of physical activity interventions in older adults with frailty or prefrailty: a systematic review and meta-analysis. CMAJ Open. 2021;9(3):E728-E43. DOI: $10.9778 /$ cmajo.20200222

20. Racey M, Ali MU, Sherifali D, Fitzpatrick-Lewis D, Lewis R, Jovkovic M, et al. Effectiveness of nutrition interventions and combined nutrition and physical activity interventions in older adults with frailty or prefrailty: a systematic review and metaanalysis. CMAJ Open. 2021;9(3):E744-E56. DOI:10.9778/cmajo.20200248 
21. Higgins JP, Altman DG, Gotzsche PC, Juni P, Moher D, Oxman AD, et al. The Cochrane Collaboration's tool for assessing risk of bias in randomised trials. BMJ. 2011;343:d5928. DOI: 10.1136/bmj.d5928

22. Schunemann H, Brozek, J., Guyatt, G., Oxman, A. The GRADE Handbook for grading the quality of evidence and the strength of recommendations. : The GRADE Working Group; 2013.

23. Woolf S, Schunemann HJ, Eccles MP, Grimshaw JM, Shekelle P. Developing clinical practice guidelines: types of evidence and outcomes; values and economics, synthesis, grading, and presentation and deriving recommendations. Implement Sci. 2012;7:61. DOI: 10.1186/1748-5908-7-61

24. Alonso-Coello P, Oxman AD, Moberg J, Brignardello-Petersen R, Akl EA, Davoli M, et al. GRADE Evidence to Decision (EtD) frameworks: a systematic and transparent approach to making well informed healthcare choices. 2: Clinical practice guidelines. BMJ. 2016;353:i2089. DOI: 10.1016/j.gaceta.2017.03.008

25. de Jong N, Chin APMJ, de Graaf C, van Staveren WA. Effect of dietary supplements and physical exercise on sensory perception, appetite, dietary intake and body weight in frail elderly subjects. Br J Nutr. 2000;83(6):605- 13. DOI: 10.1017/ s0007114500000775

26. Latham NK, Anderson CS, Lee A, Bennett DA, Moseley A, Cameron ID, et al. A randomized, controlled trial of quadriceps resistance exercise and vitamin D in frail older people: the Frailty Interventions Trial in Elderly Subjects (FITNESS). J Am Geriatr Soc. 2003;51(3):291-9. DOI: 10.1046/j.1532-5415.2003.51101.x

27. Kim CO, Lee KR. Preventive effect of protein-energy supplementation on the functional decline of frail older adults with low socioeconomic status: a communitybased randomized controlled study. J Gerontol A Biol Sci Med Sci. 2013;68(3):30916. DOI: $10.1093 /$ gerona/gls 167

28. Tieland M, van de Rest O, Dirks ML, van der Zwaluw N, Mensink M, van Loon $\mathrm{LJ}$, et al. Protein supplementation improves physical performance in frail elderly people: a randomized, double-blind, placebo-controlled trial. J Am Med Dir Assoc. 2012;13(8):720-6. DOI: 10.1016/j.jamda.2012.05.020

29. Ng TP, Feng L, Nyunt MS, Feng L, Niti M, Tan BY, et al. Nutritional, Physical, Cognitive, and Combination Interventions and Frailty Reversal Among Older Adults: A Randomized Controlled Trial. Am J Med. 2015;128(11):1225-36 e1. DOI: 10.1016/j.amjmed.2015.06.017

30. Niccoli S, Kolobov A, Bon T, Rafilovich S, Munro H, Tanner K, et al. Whey Protein Supplementation Improves Rehabilitation Outcomes in Hospitalized Geriatric Patients: A Double Blinded, Randomized Controlled Trial. J Nutr Gerontol Geriatr. 2017;36(4):149-65. DOI: 10.1080/21551197.2017.1391732

31. Park Y, Choi JE, Hwang HS. Protein supplementation improves muscle mass and physical performance in undernourished prefrail and frail elderly subjects: a randomized, double-blind, placebo-controlled trial. Am J Clin Nutr. 2018;108(5):1026-33. DOI: 10.1093/ajen/nqy214

32. Wu SY, Hsu LL, Hsu CC, Hsieh TJ, Su SC, Peng YW, et al. Dietary education with customised dishware and food supplements can reduce frailty and improve mental well-being in elderly people: A single-blind randomized controlled study. Asia Pac J Clin Nutr. 2018;27(5):1018-30. DOI: 10.6133/apjen.032018.02

33. Chen R, Wu Q, Wang D, Li Z, Liu H, Liu G, et al. Effects of elastic band exercise on the frailty states in pre-frail elderly people. Physiother Theory Pract. 2020;36(9):1000-8. DOI: 10.1080/09593985.2018.1548673

34. Losa-Reyna J, Baltasar-Fernandez I, Alcazar J, Navarro-Cruz R, Garcia- Garcia FJ, Alegre LM, et al. Effect of a short multicomponent exercise intervention focused on muscle power in frail and pre frail elderly: A pilot trial. Exp Gerontol. 2019;115:11421. DOI: $10.1016 /$ j.exger.2018.11.022

35. Liu Z, Hsu FC, Trombetti A, King AC, Liu CK, Manini TM, et al. Effect of 24-month physical activity on cognitive frailty and the role of inflammation: the LIFE randomized clinical trial. BMC Med. 2018;16(1):185. DOI: 10.1186/s12916-018 1174-8

36. Clegg A, Barber S, Young J, Iliffe S, Forster A. The Home-based Older People's Exercise (HOPE) trial: a pilot randomised controlled trial of a home-based exercise intervention for older people with frailty. Age Ageing. 2014;43(5):687-95. DOI: 10.1093/ageing/afu033

37. Yamada M, Arai H. Self-Management Group Exercise Extends Healthy Life Expectancy in Frail Community-Dwelling Older Adults. Int J Environ Res Public Health. 2017;14(5). DOI: 10.3390/ijerph14050531

38. Liu JY, Lai CK, Siu PM, Kwong E, Tse MM. An individualized exercise programme with and without behavioural change enhancement strategies for managing fatigue among frail older people: a quasi-experimental pilot study. Clin Rehabil. 2017;31(4):521-31. DOI: 10.1177/0269215516649226

39. Yoon DH, Lee JY, Song W. Effects of Resistance Exercise Training on Cognitive Function and Physical Performance in Cognitive Frailty: A Randomized Controlled Trial. J Nutr Health Aging. 2018;22(8):944-51. DOI: 10.1007/s12603-018-1090-9

40. Takatori K, Matsumoto D, Nishida M, Matsushita S, Noda T, Imamura T. Benefits of a novel concept of home-based exercise with the aim of preventing aspiration pneumonia and falls in frail older women: a pragmatic controlled trial. BMJ Open Sport Exerc Med. 2016;2(1):e000127. DOI: 10.1136/bmjsem-2016-000127

41. Tarazona-Santabalbina FJ, Gomez-Cabrera MC, Perez-Ros P, Martinez- Arnau FM, Cabo H, Tsaparas K, et al. A Multicomponent Exercise Intervention that Reverses Frailty and Improves Cognition, Emotion, and Social Networking in the CommunityDwelling Frail Elderly: A Randomized Clinical Trial. J Am Med Dir Assoc. 2016;17(5):426-33. DOI: 10.1016/j.jamda.2016.01.019

42. Kuo M-C, Chen C-M, Jeng C. A Randomized Controlled Trial of the Prescribed Stepper Walking Program in Preventing Frailty Among the Dwelling Elderly:
Application of Comprehensive Geriatric Assessment. Topics in Geriatric Rehabilitation. 2018;34(3):223-333. DOI: 10.1097/TGR.0000000000000198

43. Kwon J, Yoshida Y, Yoshida H, Kim H, Suzuki T, Lee Y. Effects of a combined physical training and nutrition intervention on physical performance and healthrelated quality of life in prefrail older women living in the community: a randomized controlled trial. J Am Med Dir Assoc. 2015;16(3):263 e1-8. DOI: 10.1016/j. jamda.2014.12.005

44. Tieland M, Verdijk LB, de Groot LC, van Loon LJ. Handgrip strength does not represent an appropriate measure to evaluate changes in muscle strength during an exercise intervention program in frail older people. Int J Sport Nutr Exerc Metab. 2015;25(1):27-36. DOI: https://doi.org/10.1123/ijsnem.2013-0123

45. Gine-Garriga M, Guerra M, Pages E, Manini TM, Jimenez R, Unnithan VB The effect of functional circuit training on physical frailty in frail older adults: a randomized controlled trial. J Aging Phys Act. 2010;18(4):401-24. DOI: 10.1123/ japa.18.4.401

46. Faber MJ, Bosscher RJ, Chin APMJ, van Wieringen PC. Effects of exercise programs on falls and mobility in frail and pre-frail older adults: A multicenter randomized controlled trial. Arch Phys Med Rehabil. 2006;87(7):885-96. DOI: 10.1016/j. apmr.2006.04.005

47. Binder EF, Schechtman KB, Ehsani AA, Steger-May K, Brown M, Sinacore DR, et al. Effects of exercise training on frailty in community-dwelling older adults: results of a randomized, controlled trial. J Am Geriatr Soc. 2002;50(12):1921-8. DOI: 10.1046/j.1532-5415.2002.50601.x

48. Gill TM, Baker DI, Gottschalk M, Peduzzi PN, Allore H, Byers A. A program to prevent functional decline in physically frail, elderly persons who live at home. $\mathrm{N}$ Engl J Med. 2002;347(14):1068-74. DOI: 10.1056/NEJMoa020423

49. Brown M, Sinacore DR, Ehsani AA, Binder EF, Holloszy JO, Kohrt WM. Lowintensity exercise as a modifier of physical frailty in older adults. Arch Phys Med Rehabil. 2000;81(7):960-5. DOI: 10.1053/apmr.2000.4425

50. Yarasheski KE, Pak-Loduca J, Hasten DL, Obert KA, Brown MB, Sinacore DR. Resistance exercise training increases mixed muscle protein synthesis rate in frail women and men >/=76 yr old. Am J Physiol. 1999;277(1):E118-25. DOI: 10.1152/ ajpendo.1999.277.1.E118

51. Yamada M, Arai H, Sonoda T, Aoyama T. Community-based exercise program is cost-effective by preventing care and disability in Japanese frail older adults. J Am Med Dir Assoc. 2012;13(6):507-11. DOI: 10.1016/j.jamda.2012.04.001

52. Chandler JM, Duncan PW, Kochersberger G, Studenski S. Is lower extremity strength gain associated with improvement in physical performance and disability in frail, community-dwelling elders? Arch Phys Med Rehabil. 1998;79(1):24-30. DOI: 10.1016/s0003-9993(98)90202-7

53. Tsang HW, Lee JL, Au DW, Wong KK, Lai KW. Developing and testing the effectiveness of a novel health qigong for frail elders in Hong Kong: a preliminary study. Evid Based Complement Alternat Med. 2013. DOI: 10.1155/2013/827392

54. Daniel K. Wii-hab for pre-frail older adults. Rehabil Nurs. 2012;37(4):195- 201. DOI: $10.1002 /$ rnj. 25

55. Gine-Garriga M, Guerra M, Unnithan VB. The effect of functional circuit training on self-reported fear of falling and health status in a group of physically frail older individuals: a randomized controlled trial. Aging Clin Exp Res. 2013;25(3):329-36 DOI: $10.1007 / \mathrm{s} 40520-013-0048-3$

56. Chen KM, Chen MH, Lin MH, Fan JT, Lin HS, Li CH. Effects of yoga on sleep quality and depression in elders in assisted living facilities. J Nurs Res. 2010;18(1):53-61. DOI: 10.1097/JNR.0b013e3181ce5189

57. Serra-Prat M, Sist X, Domenich R, Jurado L, Saiz A, Roces A, et al. Effectiveness of an intervention to prevent frailty in pre-frail community-dwelling older people consulting in primary care: a randomised controlled trial. Age Ageing. 2017;46(3):401-7. DOI: 10.1093/ageing/afw242

58. Luger E, Dorner TE, Haider S, Kapan A, Lackinger C, Schindler K. Effects of a Home-Based and Volunteer-Administered Physical Training, Nutritional, and Social Support Program on Malnutrition and Frailty in Older Persons: A Randomized Controlled Trial. J Am Med Dir Assoc. 2016;17(7):671 e9- e16. DOI: 10.1016/j. jamda.2016.04.018

59. Tieland M, Dirks ML, van der Zwaluw N, Verdijk LB, van de Rest O, de Groot $\mathrm{LC}$, et al. Protein supplementation increases muscle mass gain during prolonged resistance-type exercise training in frail elderly people: a randomized, double-blind, placebo-controlled trial. J Am Med Dir Assoc. 2012;13(8):713-9. DOI: 10.1016/j. jamda.2012.05.020

60. Kang L, Gao Y, Liu X, Liang Y, Chen Y, Liang Y, et al. Effects of whey protein nutritional supplement on muscle function among community-dwelling frail older people: A multicenter study in China. Arch Gerontol Geriatr. 2019;83:7-12. DOI 10.1016/j.archger.2019.03.012

61. Nykanen I, Rissanen TH, Sulkava R, Hartikainen S. Effects of individual dietary counseling as part of a comprehensive geriatric assessment (CGA) on nutritional status: a population-based intervention study. J Nutr Health Aging. 2014;18(1):54-8. DOI: $10.1007 / \mathrm{s} 12603-013-0342-\mathrm{y}$

62. Yamada M, Arai H, Yoshimura K, Kajiwara Y, Sonoda T, Nishiguchi S, et al Nutritional Supplementation during Resistance Training Improved Skeletal Muscle Mass in Community-Dwelling Frail Older Adults. J Frailty Aging. 2012;1(2):64-70 DOI: $10.14283 /$ jfa.2012.12

63. Clark RE, McArthur C, Papaioannou A, Cheung AM, Laprade J, Lee L, et al. «I do not have time. Is there a handout I can use?»: combining physicians' needs and behavior change theory to put physical activity evidence into practice. Osteoporos Int 2017;28(6):1953-63. DOI: 10.1007/s00198-017-3975-6 
64. Castell MV, Gutierrez-Misis A, Sanchez-Martinez M, Prieto MA, Moreno B, Nunez $\mathrm{S}$, et al. Effectiveness of an intervention in multicomponent exercise in primary care to improve frailty parameters in patients over 70 years of age (MEFAP-project), a randomised clinical trial: rationale and study design. BMC Geriatr. 2019;19(1):25. DOI: $10.1186 / \mathrm{s} 12877-018-1024-8$

65. Baert V, Gorus E, Guldemont N, De Coster S, Bautmans I. Physiotherapists' perceived motivators and barriers for organizing physical activity for older long-term care facility residents. J Am Med Dir Assoc. 2015;16(5):371-9. DOI: 10.1016/j. jamda.2014.12.010

66. Dent E, Morley JE, Cruz-Jentoft AJ, Woodhouse L, Rodriguez-Manas L, Fried LP, et al. Physical Frailty: ICFSR International Clinical Practice Guidelines for Identification and Management. J Nutr Health Aging. 2019;23(9):771-87. DOI: $10.1007 / \mathrm{s} 12603-019-1273-\mathrm{Z}$
67. Tomasone JR, Flood SM, Latimer-Cheung AE, Faulkner G, Duggan M, Jones R, et al. Knowledge translation of the Canadian 24-Hour Movement Guidelines for Adults aged 18-64 years and Adults aged 65 years or older: a collaborative movement guideline knowledge translation process. Appl Physiol Nutr Metab. 2020;45(10 (Suppl. 2)):S103-S24. DOI: 10.1139/apnm-2020-0601

68. Muscedere J, Andrew MK, Bagshaw SM, Estabrooks C, Hogan D, Holroyd-Leduc J, et al. Screening for Frailty in Canada's Health Care System: A Time for Action. Can J Aging. 2016;35(3):281-97. DOI: 10.1017/S0714980816000301

69. Muscedere J, Afilalo J, Araujo de Carvalho I, Cesari M, Clegg A, Eriksen HE, et al. Moving Towards Common Data Elements and Core Outcome Measures in Frailty Research. J Frailty Aging. 2020;9(1):14-22. DOI: 10.14283/jfa.2019.43

How to cite this article: A.L. Lorbergs, J.C. Prorok, J. Holroyd-Leduc, et al. Nutrition and Physical Activity Clinical Practice Guidelines for Older Adults Living with Frailty. J Frailty Aging 2022;11(1)3-11; http://dx.doi.org/10.14283/jfa.2021.51 